\title{
Tunneled and routine peripherally inserted central catheters placement in adult and pediatric population: review, technical feasibility, and troubleshooting
}

\author{
Nishant Gupta ${ }^{1}$, Darshan Gandhi ${ }^{2}$, Salil Sharma ${ }^{1}$, Pradeep Goyal ${ }^{3}$, Gagandeep Choudhary ${ }^{4}$, Shuo Li $^{5}$ \\ ${ }^{1}$ Department of Radiology, Bassett Healthcare, Cooperstown, NY, USA; ${ }^{2}$ Department of Radiology, Northwestern Memorial Hospital, Northwestern \\ University Feinberg School of Medicine, Chicago, IL, USA; ${ }^{3}$ Department of Radiology, University of Rochester Medical Center, Rochester, NY, \\ USA; ${ }^{4}$ Department of Radiology; Division of Molecular Imaging \& Therapeutics and Neuroradiology, University of Alabama at Birmingham, \\ Birmingham, AL, USA; ${ }^{5}$ Department of Radiology, KU School of Medicine-Wichita, University in Wichita, Wichita, KS, USA
}

Correspondence to: Darshan Gandhi, MD. Department of Radiology, Northwestern Memorial Hospital, Northwestern University Feinberg School of Medicine, 676 N St. Clair St, Suite 800, Chicago, IL 60611, USA. Email: darshangandhi7@gmail.com.

\begin{abstract}
Vascular access procedures are crucial for the management of various critically ill pediatric and adult patients. Venous access is commonly performed in the form routine as well as tunneled peripherally inserted central catheters (PICC). These venous accesses are commonly used in emergency, surgical as well as ICU settings, for various infusions, total parenteral nutrition, long term intravenous antibiotics, frequent blood draws, etc. PICC insertion is guided using ultrasound and fluoroscopic guidance, which decreases the risk of complications that are otherwise seen with central venous accesses like triple lumen catheters, etc. PICC insertion and care is very simple and can be performed by specially trained PICC nurses and that helps in decreasing the overall cost of healthcare. This review article is written with educational intent for the readers to discuss indications, contraindications, procedure techniques, imaging, care of routine as well as tunneled PICC.
\end{abstract}

Keywords: Peripherally inserted central catheters (PICC); pediatric; adult; ultrasound

Submitted May 25, 2020. Accepted for publication Oct 28, 2020.

doi: 10.21037/qims-20-694

View this article at: http://dx.doi.org/10.21037/qims-20-694

\section{Introduction}

Peripheral inserted central venous catheter (PICC) placement is the most common vascular interventional procedure performed in both adults and pediatric patients. As the name suggests the catheter is inserted in a peripheral vein and advanced so that its tip lies in central venous system. Ultrasound guidance has shown a better rate of success (1).

\section{Indications}

PICC is used for infusion of medications over prolonged period of time and total parenteral nutrition (TPN). The medications include but not limited to antibiotics and chemotherapeutic agents. PICC is favored when the course of infusion is 14 days or greater but less than 3 months $(2,3)$. It is useful in premature neonates (4) and in patients who receive frequent phlebotomy (5). Placement of PICC in burns patients early in the course of treatment decreases risk of bacteremia $(5,6)$. Patients can be discharged form hospital with PICC lines for completion of intravenous therapy at home or nursing homes $(5,6)$. PICC can be used as a temporary measure in patients who need chronic or lifelong access (sickle cell, cystic fibrosis, short gut) or frequently hospitalized, as tunneled central venous catheter is preferred method in that patient population. 


\section{Preprocedural evaluation and planning}

In patients needing PICC placement, the clinical information is reviewed by a member of the interventional radiology (IR) team. Occasionally, in case of previous history of difficult access in adult patients and in all pediatric patients, alternate forms of venous access use, such as central venous catheter, typically through jugular or femoral approach, should also be discussed with the referring team, patient and/or family during the consent.

Presence of altered venous anatomy from congenital or acquired conditions can pose technical difficulties during PICC placement. Congenital anomalies include situs anomalies, duplicated superior vena cava (SVC) or left-sided SVC, and the presence of surgical shunts (e.g., Glenn cavopulmonary shunt). Therefore, detailed review of surgical history and previous imaging is essential. If the procedure is planned bedside, then the guidance may be obtained using electromagnetic method/EKG or Sherlock method for tip localization. If done in IR suite then fluoroscopic guidance is ideal.

It is important to review history of PICC placements as chronic venous injury and stenosis is a frequent complication especially in patients with history of multiple PICCs. Detailed inquiry should be made regarding which extremity and vein was utilized and if there was any difficulty in placement. Use of alternate extremities should be done in those who need repeat procedure, to decrease the risk of injury and stenosis (7).

Medical history of coagulopathy or anticoagulant or antiplatelet therapy poses increased risk of bleeding complications. Optimizing the coagulation profile before performing the procedures is required. International normalized ratio (INR) should be $<2.0$, INR $>2.0$ requires treatment (i.e., FFP, vitamin K). Minimum platelet count requirement of $20,000 / \mathrm{L}$, with blood transfusion recommended for counts below 20,000/L. Clopidogrel should be withheld for 5 days before the procedure and for Low molecular weight heparin (LMWH) withhold one therapeutic dose before the procedure, and there is no need to withhold aspirin for PICCs (8). Apart from medical measures, using ultrasound guidance and smallest gauge needle (optimally with a single pass) for peripheral venous access can significantly reduce bleeding risk, especially in high risk patients. Ultrasound is also useful post procedure to evaluate for bleeding and hematoma formation (8).

Finally, the most important pre-procedure step is to obtain informed consent from patient or legally responsible authority. This includes explaining the procedure, complications, alternatives to the procedure as well as answering to their queries.

\section{Sedation}

Sedation is indicated only in pediatric patients or in adults with significant anxiety or altered senses. In infants and young children, sedation with IV midazolam and ketamine for both non-tunneled and tunneled PICC may be used. Lorazepam 1-2 mg oral/liquid dose with or without hydromorphone hydrochloride $2-4 \mathrm{mg}$ oral/liquid doses is used for non-tunneled PICC in adult. Whereas moderate sedation with IV fentanyl and midazolam may be used for tunneled PICCs placement in almost all cases. Cardiopulmonary monitoring by a skilled nurse or nurse anesthetist is required during the procedure where moderate sedation is used. In some cases, and in some countries, monitored anesthesia care is required to assist with PICC placement. It For intensive care unit (ICU) patients undergoing PICC placement in IR suites, the presence of ICU nurse during the procedure is paramount to assist in the monitoring and clinical care of the patient.

\section{Patient positioning}

Patient is positioned supine on procedure table with arm abducted and hand supinated and supported on arm board extension. Please see the table setup as in Figure 1. The arm is fastened firmly to the board, draped, and scrubbed from forearm to axilla. Lower extremities can also be fastened. Sterile scrub and full gown and glove protocol are followed by the performing physician.

\section{Vein selection and planning}

A tourniquet is applied proximally in the upper extremity for adequate distention of veins without compromising the arterial blood flow (9). Preliminary ultrasound (linear 7-12 MHz transducer for adult and 15-18 MHz hockey stick transducer for infants and young children) is used to map the available veins and assess their patency and compressibility. The selected vein is accessed in a transverse short-axis section to avoid potential arterial puncture, especially if accessing brachial vein. PICC size selection is based on the size of the vein, for example, if the vein is $5 \mathrm{~mm}$ in diameter then a $5 \mathrm{Fr}$ PICC will be most suitable.

The first attempt at venipuncture should be as distal as 


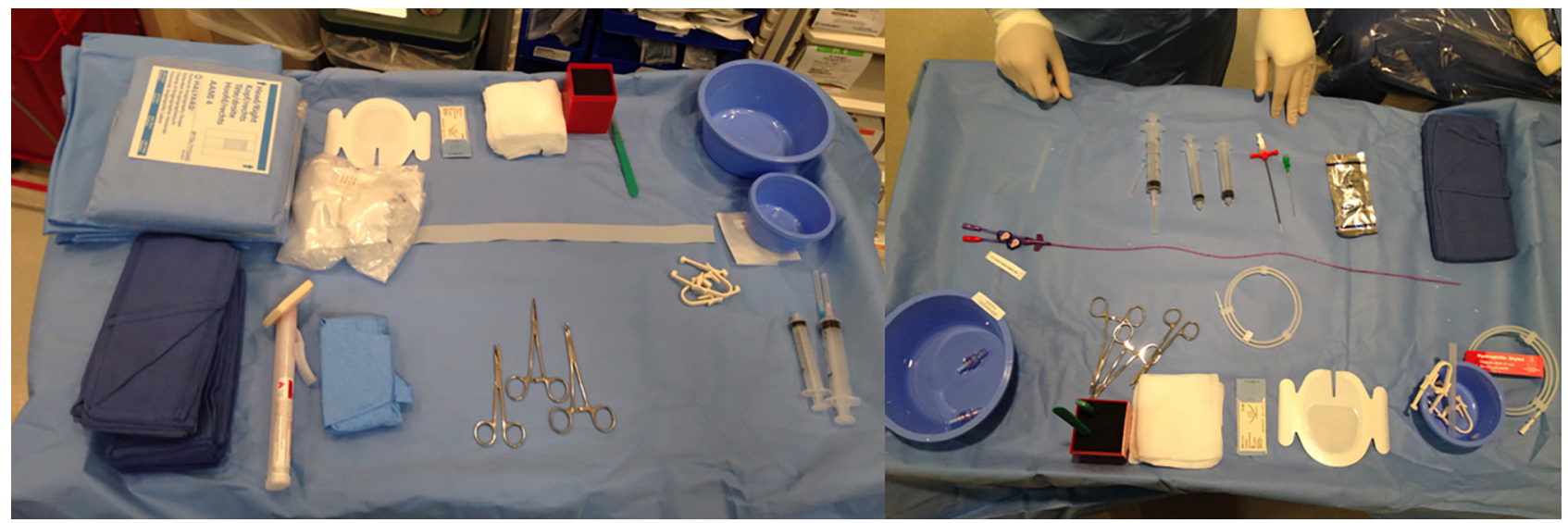

Figure 1 Table setup —usual table setup for PICC placement. PICC, peripherally inserted central catheters.

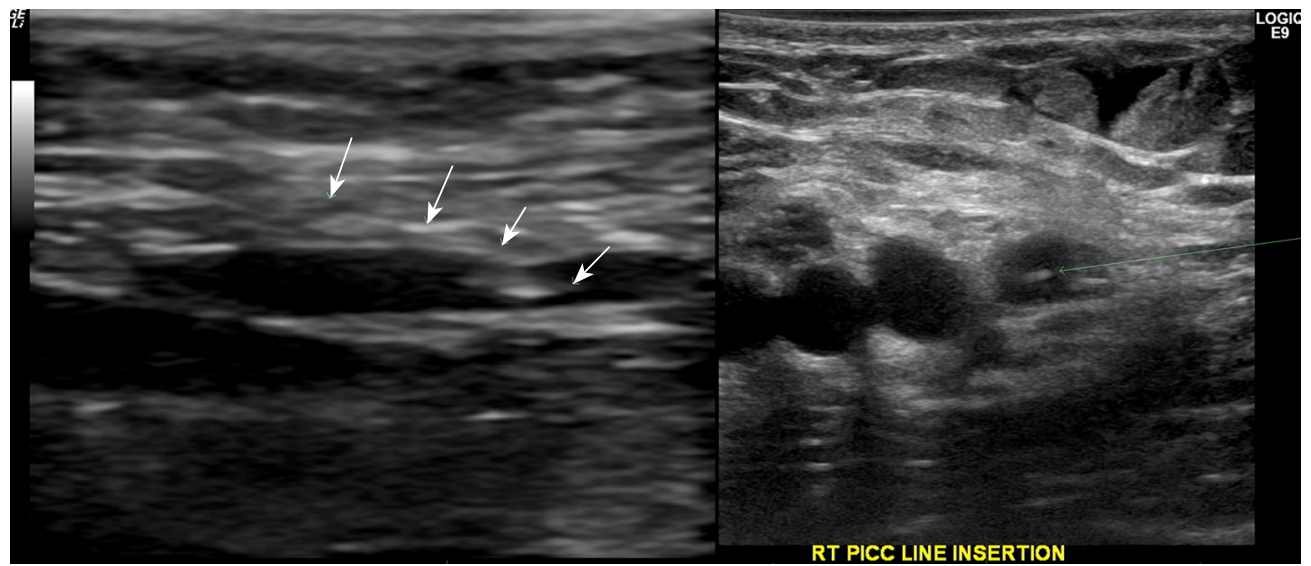

Figure 2 The angle of the needle relative to the arm should be $<45^{\circ}$. The arrows showed the needle and its angle of entry in the vessel.

possible above the antecubital fossa. Antecubital fossa access should be avoided as it causes post procedure discomfort to the patient. If the first attempt of venous access fails, further attempts can be made in mid and proximal arm, proximal to the vein injury. A patent, sizeable basilic vein is always preferred for initial access attempt. Basilic vein can be easily identified in medial arm as it is very superficial.

The second preference is the brachial vein. It can be single or paired veins adjacent to brachial artery. It lies deeper and often in close proximity to the median nerve. Although the incidence is rare, care should be taken to avoid inadvertent arterial puncture and injury to the median nerve during brachial vein access (10). Cephalic vein is less preferred due to multiple reasons. It is often small, demonstrates high incidence of anatomical variation in the course, presence of acute angulation in its course in axilla and association with higher incidences of thrombosis makes cephalic vein the least favorite. Extensive training and experience are required in ultrasound-guided venipuncture in infants and young children due to small caliber of veins.

\section{Venipuncture and PICC placement}

The skin at the chosen site of venous access is marked with marker pen. Very small amount of $1 \%$ lidocaine is infiltrated superficially to avoid venospasm with a 25 -gauge needle. For venipuncture, a 20-G (22-G and 24-G for children and infant respectively) sheathed Angiocath IV catheter (Surflo Teflon IV catheters, Terumo Medical Corporation, Somerset, NJ) or 21-G echo tip needle 5 or $7 \mathrm{~cm}$ (Cook Medical, Bloomington, IN) depending on the depth of vein is used and the catheter is positioned with the bevel up at an angle of 45 degrees (Figure 2). It is important that the course of needle tip is visible on ultrasound as the needle 
tip enters the superficial wall of the vein. The tip or shaft of needle appear as an echogenic dot on ultrasound screen. In order to localize the tip of needle, the ultrasound probe is swept away form the needle gently, while keeping the needle stationary until the needle is no longer visualized. The ultrasound probe is then swept towards the needle until the needle tip is visualized, confirming tip position. Once the tip of needle is positioned directly over the superficial wall in the middle of vessel, the needle is advanced to puncture the wall and enter the lumen. This entry image is saved.

There are two ways to access a vein: the single-wall puncture and the double-wall puncture technique. In single wall puncture, only superficial wall is punctured. The needle hub is flattened to make the entry angle shallow to avoid injury to deeper wall. With this technique there is minimal damage to vein and no hematoma formation, making it an ideal approach in patients with high risk of bleeding. Another advantage is that the needle can be carefully threaded into the venous lumen, making it easier to follow with a guidewire. The biggest drawback with this technique is that the entry angle becomes shallow, which means use of longer length needle as the vein puncture site will be distant from skin entry site. This technique definitely requires more skill and expertise. In double-wall puncture technique, a needle is used to puncture both superficial and deep walls using a relatively steeper angle. Irrespective of the technique, the angle of needle relative to skin should always be $<45^{\circ}$ (Figure 1). If the needle is angled too steep then the guidewire inserted through the needle hits the deeper/ posterior wall of the vein giving resistance or it may even push the needle out of the venous lumen.

A series of few screwing longitudinal advances is made to thread the needle tip and gain stable venous purchase. If this step is foregone, the needle tip could be placed within the vein while the sheath remains in the soft tissues. Care is taken to maintain the tip in the center of the vein lumen. Once the Angiocath is advanced fully with its hub flush with the skin, blood can be seen returning from the hub confirming adequate intraluminal placement. The inner stylet is then removed, leaving the angiocath in place and upper arm tourniquet is released. A 0.018" platinum guidewire is then advanced through the angiocath under intermittent fluoroscopic guidance to make sure that the wire goes toward the right side of heart. Wire going to left side of heart suggests arterial puncture or left sided superior vena cava (SVC) with wire coursing in coronary sinus, which is rare. Confirmation of placement in venous side can be done by advancing the wire in inferior vena cava (IVC) or injecting small amount of contrast.

An 0.018-inch guidewire is used for adults and older children in which 20,21 or $22 \mathrm{G}$ needle is used but for children where smaller than 22-G needle is used, thinner wire is used as $24 \mathrm{G}$ needle does not accept a 0.018 "diameter guidewire. In pediatric patients, thinner guidewires (few, e.g., 0.010", 60-cm Nitinol Mandrel Wire (Galt Medical, Garland, TX); 0.014", 50-cm Floppy II Hi-Torque Guidewire (Abbott Vascular, Santa Clara, CA), are used to facilitate access.

Once the guidewire is in place, small amount of lidocaine is infiltrated in deeper subcutaneous tissue and a small skin incision is made with a blade. The track is dilated with inner dilator of peel-away sheath. The skin and soft tissues of arm adjacent to puncture are stretched to provide tissue support. A peel-away sheath (typically $0.5-1 \mathrm{Fr}$ larger in diameter than the PICC catheter itself) is included in most imageguided PICC sets, however it is often not needed. Its use should be avoided in young children and infants, as there is increased post insertion oozing. With adequate dilatation of track using the inner dilator, the cut to length catheter can be passed over the wire through the subcutaneous tissues into the vein in most cases with minimal resistance. Some of the initial resistance can also be overcome by advancing both wire and catheter together for a short distance. Once there is no resistance the wire is pinched in place and catheter is advanced over the wire into the SVC at the level or just above the Superior Cavo-atrial junction using intermittent fluoroscopic guidance. If the initial standard wire provided in the kit cannot be advanced centrally, then contrast is injected through the dilator and venogram is performed. A stenosis or collateral vessel ca be manipulated using an 0.018 " hydrophilic wire.

Once the wire tip is in the required position, it is clamped with a hemostat just outside the hub of dilator or sheath. The wire is withdrawn and its length from tip to clamp is used for PICC length measurement. A saline syringe is used to flush the sheath and cap the sheath to avoid air embolism. The length of PICC cut to the size of wire is documented in patient's chart. Precise measurement is required in infants as the difference of a few millimeters can be quite significant as the tip position changes with arm position (11). In infants and young children, a provocative adduction maneuver can be performed at the time of wire measurement and/or final PICC position to check the migration of PICC tip. If the tip descends in the right atrium with the maneuver, then it needs to be shortened to the appropriate length. A fluoroscopic spot image is 

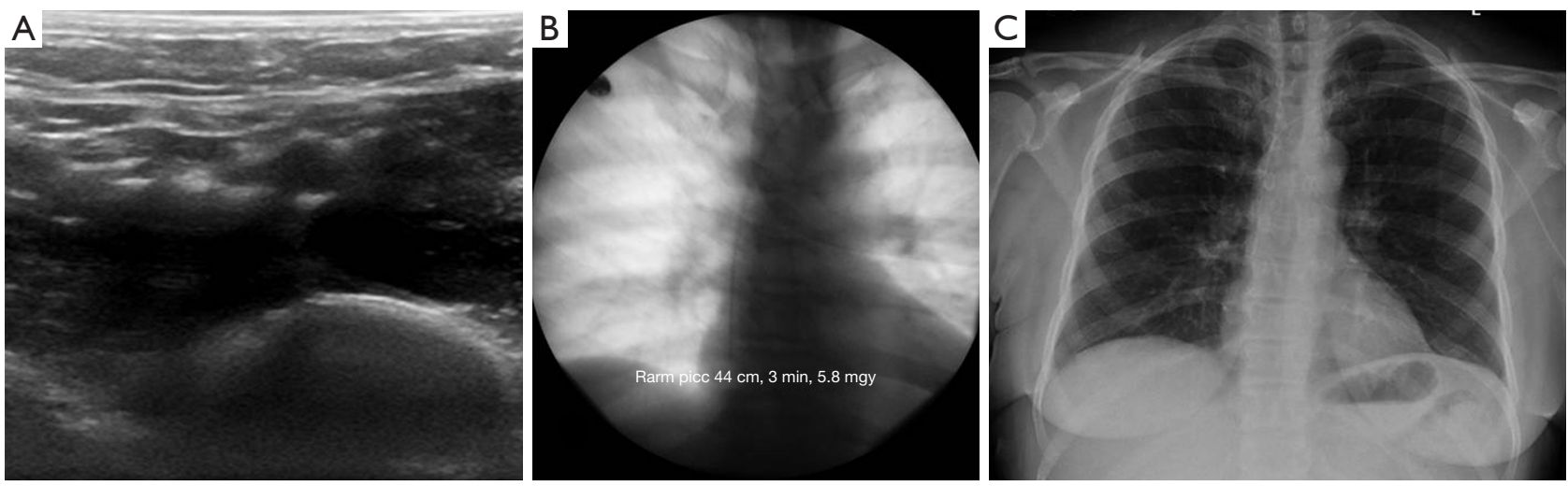

Figure 3 Sequential events in a typical PICC line placement. (A) A transverse sonographic image of the basilic vein in an adult patient; (B) the tip of the catheter at RA-SVC junction; (C) the final routine PICC placement radiograph in an adult patient requiring long term iv access. PICC, peripherally inserted central catheters.

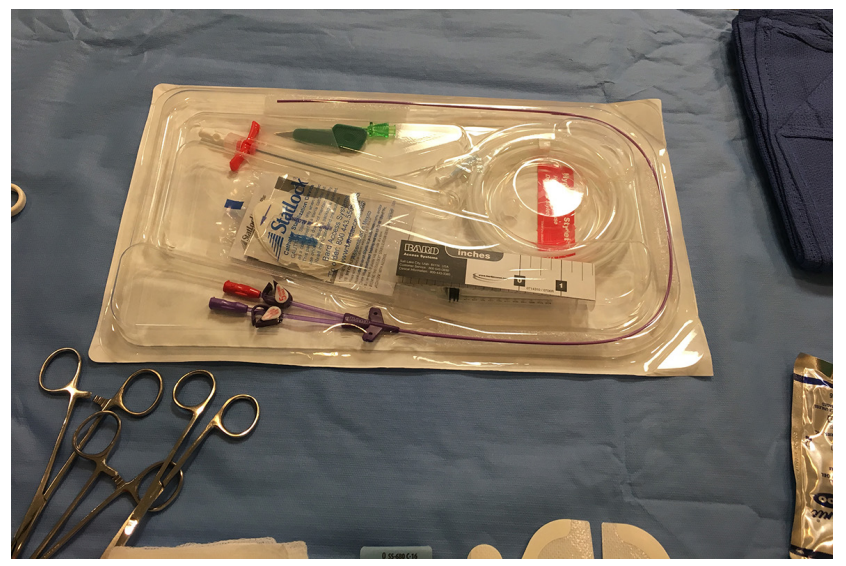

Figure 4 This is a typical adult kit. The PICC is a power-PICC meaning it can be used while performing CT for power injection for CTA. PICC, peripherally inserted central catheters; CTA, computed tomography angiography.

saved to document the adequate position of tip (Figure 3), at the superior Cavo-atrial junction if inserted through the arm and inferior Cavo-atrial junction, if inserted through the femoral vein. Other available methods for tip localization are Sherlock tip localization and ECG based localization techniques. Intracavitary echocardiography for tip localization has also been described as a noninvasive method with high accuracy (4). A good rule of thumb is that superior Cavo-atrial junction is anatomically located two vertebral bodies below the carina on a frontal chest radiograph (Figure 3). A few exceptions exist for the catheter tip position of arm PICC. The tip is left in medial subclavian vein in children with functional single ventricle who have a superior cavopulmonary connection (bidirectional Glenn or hemi-Fontan operation). The position of the catheter tip near the pulmonary arteries causes increased risk of life-threatening thrombosis in these patients. Similarly, pediatric patients with right atrial irritability and those with dysrhythmia require shorter PICC with the tip higher than the conventional position.

Once the PICC is on place and lumens checked for good return, the hub is secured to skin with adhesive device (e.g., StatLock; Bard, Covington, GA) in children. The lumen is flushed with heparin solution and capped. An antimicrobial disc is placed on the skin entry site followed by a clear adhesive covering.

There are several PICCs available differing in sizes (Figure 4) and materials, and their description is beyond the scope of this article, however, a few points are worth mentioning. Small caliber PICC are used in infants and 
young children as the veins are narrow, therefore single lumen PICC are preferred, unless there is a specific indication for additional lumen. Most often a 3-Fr singlelumen in children and $5 \mathrm{Fr}$ single-lumen catheter in adult (Cook, Bloomington, IN) is preferred. Lines of smaller caliber (1.9 Fr single-lumen and 2.6 Fr double lumen) are used in infants and neonates. The material of PICC is also important. A soft pliable catheter made of silicon is used in young children to reduce the potential risk of vascular rupture or cardiac tamponade. Dual lumen and/or power injectable polyurethan PICCs are used in older children and adults. The position of tip in SVC or Cavo-atrial junction cannot be more emphasized as there are reported cases of fatal cardiac tamponade with atrial perforation from catheter tip in right atrium $(12,13)$.

In post-operative pediatric patients with congenital heart disease it is desirable to place the tip in subclavian vein to avoid shunt thrombosis. It is imperative to communicate the position with pediatric cardiac services in advance to avoid malposition and revision in these patients.

\section{Technique for tunneled femoral PICC line}

Tunneled internal jugular (IJ) or femoral vein approach PICC lines are crucial in patients with failed arm venous access, veins too small in caliber relative to the size of the catheter and in children with chronic renal disease where the arm veins are preserved for possible future requirement of creating a fistula for hemodialysis. A standard PICC catheter is placed after obtaining access from IJ or femoral vein (or saphenous vein).

Studies have shown increased rate of line sepsis or infection with femoral vein access in groin $(14,15)$. A modified technique for tunneled femoral PICC can be used where the skin entry site in the distal medial thigh near the femoral condyles, thereby reducing the increased risk of line infection with groin skin entry site. The access needle is passed cranially to puncture the femoral vein at a safe site in the groin region. This technique requires a relatively long needle such as a $21 \mathrm{G}, 5$ - or 7 -cm needle because of more distance to be traversed in soft tissue before venous access. The access needle is used to create a long shallow tunnel in the subcutaneous tissues using ultrasound guidance before puncture of the common femoral vein. Another technique is using the peel-away sheath to create a tunnel as in conventional fashion and the PICC os advanced through it to the vessel entry site. Compression above the inguinal fold with fourth and fifth fingers, while holding the ultrasound probe with thumb and index finger causes distention of common femoral vein easing the access. Once venous access is obtained, the catheter is placed in usual fashion using a standard Seldinger technique. The tip of catheter is positioned at superior Cavo-atrial junction in IJ approach and inferior Cavo-atrial junction femoral approach.

\section{Tips and tricks}

* In infants and young children, the soft tissues are mobile and tend to push away from needles during venipuncture. This can be minimized by getting intrafascial position of needle tip and angiocatheter sheath before venipuncture and then advancing the needle with firm, yet controlled, small screwing movements. There is an initial "blood flash" at the hub as the metal needle tip enters the lumen, however it should be ignored as it does not mean that the catheter is in the lumen. The needle catheter assembly should be advanced while monitoring the needle tip position with ultrasound, until a second "pop" is felt, which signifies that the catheter has entered the lumen. At this point the needle is carefully withdrawn until blood return appears and then attempt is made to pass the guidewire. If the second "pop" is not felt, the needle is hubbed on the skin and attempt is made to advance the angiocath up. If it can be advanced for a substantial distance (approximately $1 \mathrm{~cm}$ ) without resistance, it means that the catheter in inside the lumen.

* In poorly hydrated Infants vascular access can be unusually difficult. In these cases, a superficial peripheral vein of arm or leg is accessed under US guidance and a fluid bolus $(5 \mathrm{~mL} / \mathrm{kg})$ is administered at the beginning of the procedure. This improves venous filling, thereby distending the lumen of basilica/ brachial veins. This improves the chances of successful venipuncture (16).

* There are instances where following a successful venipuncture the platinum tip wire cannot be advanced centrally due to venous spasm or stenosis. Whenever there is resistance in advancing the wire at any step, fluoroscopic imaging is required. Most often the venospasm at the level of axilla is related to tourniquet placement. Releasing the tourniquet and waiting for a couple minutes is helpful in such cases. Venous stenosis is another potential culprit. It can happen anywhere in the venous system and is 
frequently the cause in those with history of PICC or central venous lines. Difficulty in advancing the wire should always be dealt with gentle manipulation of initial guidewire under fluoroscopic guidance. If that fails, the initial guidewire is exchanged with a short 0.018-inch hydrophilic guidewire followed by repeat gentle manipulation. The stenosis can be negotiated with this technique in most of the cases. In resistant cases, a venogram is performed by gently injecting contrast through a short 3 or 4-Fr dilator. Venogram delineates the vascular anatomy and provides roadmap for wire manipulation. If venogram reveals complete occlusion and numerous collaterals, then the operator can decide whether to leave a short non central PICC for example, in subclavian vein or a well-developed venous collateral or to attempt access and PICC placement in the opposite extremity.

* It has been often seen in tunneled line, that the catheter is either cut shorter or longer, which needs to reposition and most of the time need to completely redo in pediatric patients. This can be overcome to some extent by making $2-3 \mathrm{~cm}$ longer subcutaneous tunnel than usual and pulling the catheter cuff towards the venotomy site initially. If catheter tip is extending into the right atrium, catheter-cuff can be pulled back in the tunnel until catheter tip located at cavoatrial junction. If the catheter falls shorter and catheter tip is in the innominate vein or proximal SVC, catheter-cuff can be pushed to as near to venotomy site as possible.

* Inadvertent puncture of carotid artery during the $\mathrm{IJ}$ access tunneled line in neonate and infant can be avoided by puncturing the IJV near the Innominate junction, so that continuation of IJV with innominate vein can be seen in the same longitudinal view by placing the hockey stick probe slight obliquely pointing under the clavicular head.

\section{Complications}

Although serious complications are rare, several complications can occur with PICC line insertions especially in children $(17,18)$. Complications can happen during placement of PICC such as arterial puncture, air embolism, injury to nerves, catheter malposition, cardiac arrhythmias, and rarely cardiac perforation with resultant fatal cardiac tamponade. Complications following placement include line infections, line migration, venous thrombosis, and stenosis. A few studies have shown up to $40 \%$ incidence of venous thrombosis after PICC line insertion (19). Bloodstream infection are also reported (20). Larger catheters are associated with increased thrombosis and use of smaller lumen PICC is associated with reduced risk $(21,22)$. A study by Sharp et al. shows that the rate of symptomatic venous thromboembolism with PICC is lower if the size of catheter is less than $45 \%$ of the venous diameter (23). Inadvertent arterial puncture can result in brachial arteriovenous fistula, pseudoaneurysm formation and potential compromised of distal arterial blood flow $(24,25)$. There should be periprocedural checklist during PICC line insertion to avoid complications like retained guidewires (26).

\section{Line management}

Line dislodgement can be avoided by securing them with adhesive device (e.g., StatLock; Bard, Covington, GA) or by sutures. As mentioned earlier the PICC line is primed with heparin after placement. The concentration of heparin varies with patient's weight. In children weighing $<15 \mathrm{~kg}$ the concentration used is $100 \mathrm{U} / \mathrm{mL}$ and in children weighing $>15 \mathrm{~kg}$ the concentration is $10 \mathrm{U} / \mathrm{mL}$. The volume of heparin to be flushed varies with the catheter type and is mentioned on the catheter lumen hub. Clot within the PICC needs administration of $1 \mathrm{mg}$ of tissue plasminogen activator, which is allowed to stay in the PICC for 1 hour.

Another concern about continuous oozing of blood from the insertion site after PICC placement, especially in patients prone to bleeding and oozing, i.e., anticoagulation, low platelets, low albumin etc., BioSeal CVC powder (Biolife LLC, Sarasota, FL) is applied at the insertion site as well at the suture site if sutures are used to secure the catheter because it absorbs the bleeding from any site. It generally eliminates the need for a 24-hour dressing.

Because infection is a prime concern, at some institute antimicrobial disk (BIOPATCH ${ }^{\circledR}$ Protective Disk with CHG, Johnson \& Johnson, Ethicon, and Somerville, $\mathrm{NJ}$ ) is applied at the time of first dressing change after 24 hours, could not be applied at time of PICC insertion due to the oozing that often occurs. Entire nursing staff needs to be educated how to handle and manage PICC lines. Aseptic precautions should be taken while handling the line including use of clean-gloved hands. Every time the line is handled the hub is thoroughly cleaned with alcohol swab allowing it to dry. A study by Rutkoff et al. showed that using antimicrobial PICC can reduce the level of infection by a factor of 4 (27). 
Any concerns related of management or functioning of PICC from clinical team needs to be communicated to an IR nurse. Appropriate management and necessary imaging or intervention can be planned accordingly by the IR team. PICC can be safely removed after the completion of treatment without difficulty. The length of removed PICC is measured and compared with the length recorded at the time of PICC insertion.

\section{Conclusions}

PICC insertion and care is very simple and can be performed by specially trained PICC nurses and that helps in decreasing the overall cost of healthcare. This review article is written with educational intent for the readers to discuss indications, contraindications, procedure techniques, imaging, care of routine as well as tunneled PICCs.

\section{Acknowledgments}

Funding: None.

\section{Footnote}

Conflicts of Interest: All authors have completed the ICMJE uniform disclosure form (available at http://dx.doi. org/10.21037/qims-20-694). The authors have no conflicts of interest to declare.

Open Access Statement: This is an Open Access article distributed in accordance with the Creative Commons Attribution-NonCommercial-NoDerivs 4.0 International License (CC BY-NC-ND 4.0), which permits the noncommercial replication and distribution of the article with the strict proviso that no changes or edits are made and the original work is properly cited (including links to both the formal publication through the relevant DOI and the license). See: https://creativecommons.org/licenses/by-nc-nd/4.0/.

\section{References}

1. Moraza-Dulanto MI, Garate-Echenique L, MirandaSerrano E, Armenteros-Yeguas V, Tomás-López MA, Benítez-Delgado B. Ultrasound-guided peripherally inserted central catheters (PICC) in cancer patients: success of the insertion, survival and complications. Enferm Clin 2012;22:135-43.

2. Chopra V, Flanders SA, Saint S, Woller SC, O'Grady
NP, Safdar N, Trerotola SO, Saran R, Moureau N, Wiseman S, Pittiruti M, Akl EA, Lee AY, Courey A, Swaminathan L, LeDonne J, Becker C, Krein SL, Bernstein SJ; Michigan Appropriateness Guide for Intravenouse Catheters (MAGIC) Panel. The Michigan Appropriateness Guide for Intravenous Catheters (MAGIC): Results From a Multispecialty Panel Using the RAND/UCLA Appropriateness Method. Ann Intern Med 2015;163:S1-40.

3. Crocoli A, Cesaro S, Cellini M, Rossetti F, Sidro L, Pinelli F, Pittiruti M. In defense of the use of peripherally inserted central catheters in pediatric patients. J Vasc Access 2020. [Epub ahead of print]. doi:10.1177/1129729820936411.

4. Pittiruti M, Pelagatti F, Pinelli F. Intracavitary electrocardiography for tip location during central venous catheterization: A narrative review of 70 years of clinical studies. J Vasc Access 2020. [Epub ahead of print]. doi: $10.1177 / 1129729820929835$.

5. Tan R, Knowles D, Streato C, Johnston AJ. The use of peripheraly inserted central catheters in intensive care: should you pick the PICC? J Intensive Care Soc 2009;10:95-8.

6. Armstrong SD, Thomas W, Neaman KC, Ford RD, Paulson J. The impact of antibiotic impregnated PICC lines on the incidence of bacteremia in a regional burn center. Burns 2013;39:632-5.

7. Duwadi S, Zhao Q, Budal BS. Peripherally inserted central catheters in critically ill patients - complications and its prevention: A review. Int J Nurs Sci 2018;6:99-105.

8. Patel IJ, Rahim S, Davidson JC, Hanks SE, Tam AL, Walker TG, Wilkins LR, Sarode R, Weinberg I. Society of Interventional Radiology consensus guidelines for the periprocedural management of thrombotic and bleeding risk in patients undergoing percutaneous image-guided interventions-Part II: recommendations. J Vasc Interv Radiol 2019;30:1168-84.e1.

9. Krishnamurthy G, Keller MS. Vascular access in children. Cardiovasc Intervent Radiol 2011;34:14-24.

10. Alomari A, Falk A. Median nerve bisection: a morbid complication of a peripherally inserted central catheter. J Vasc Access 2006;7:129-31.

11. Connolly B, Amaral J, Walsh S, Temple M, Chait P, Stephens D. Influence of arm movement on central tip location of peripherally inserted central catheters (PICCs). Pediatr Radiol 2006;36:845-50.

12. Racadio JM, Doellman DA, Johnson ND, Bean JA, Jacobs BR. Pediatric peripherally inserted central catheters: complication rates related to catheter tip location. Pediatrics 2001;107:E28. 
13. Towbin R. The bowed catheter sign: A risk for pericardial tamponade. Pediatr Radiol 2008;38:331-5.

14. Tsai MH, Lien R, Wang JW, Huang HR, Chiang CC, Chu SM, Hsu JF, Huang YC. Complication rates with central venous catheters inserted at femoral and non-femoral sites in very low birth weight infants. Pediatr Infect Dis J 2009;28:966-70.

15. Trottier SJ, Veremakis C, O'Brien J, Auer AI. Femoral deep vein thrombosis associated with central venous catheterization: results from a prospective, randomized trial. Crit Care Med 1995;23:52-9.

16. Bhutta ST, James CA, Roberson PK, Li S. Challenging infant PICCs: does a procedural fluid bolus increase vein size? Pediatr Radiol 2004;34:77.

17. Crowley JJ, Pereira JK, Harris LS, Becker CJ. Peripherally inserted central catheters: experience in 523 children. Radiology 1997;204:617-21.

18. Dubois J, Rypens F, Garel L, David M, Lacroix J, Gauvin F. Incidence of deep vein thrombosis related to peripherally inserted central catheters in children and adolescents. CMAJ 2007;177:1185-90.

19. Abdullah BJ, Mohammad N, Sangkar JV, Abd Aziz YF, Gan GG, Goh KY, Benedict I. Incidence of upper limb venous thrombosis associated with peripherally inserted central catheters (PICC). Br J Radiol 2005;78:596-600.

20. Chopra V, Ratz D, Kuhn L, Lopus T, Chenoweth C, Krein S. PICC-Associated Bloodstream Infections: Prevalence, Patterns, and Predictors. Am J Med 2014;127:319-28.

Cite this article as: Gupta N, Gandhi D, Sharma S, Goyal P, Choudhary G, Li S. Tunneled and routine peripherally inserted central catheters placement in adult and pediatric population: review, technical feasibility, and troubleshooting. Quant Imaging Med Surg 2021;11(4):1619-1627. doi: 10.21037/qims-20694
21. Grove JR, Pevec WC. Venous thrombosis related to peripherally inserted central catheters. J Vasc Interv Radiol 2000;11:837-40.

22. Evans RS, Sharp JH, Linford LH, Lloyd JF, Woller SC, Stevens SM, Elliott CG, Tripp JS, Jones SS, Weaver LK. Reduction of peripherally inserted central catheterassociated DVT. Chest 2013;143:627-33.

23. Sharp R, Cummings M, Fielder A, Mikocka-Walus A, Grech C, Esterman A. The catheter to vein ratio and rates of symptomatic venous thromboembolism in patients with a peripherally inserted central catheter (PICC): a prospective cohort study. Int J Nurs Stud 2015;52:677-85.

24. Tran HS, Burrows BJ, Zang WA, Han DC. Brachial arteriovenous fistula as a complication of placement of peripherally inserted central venous catheter: a case report and review of the literature. Am Surg 2006;72:833-6.

25. Dzepina I, Unusic J, Mijatovic D, Bulic K. Pseudoaneurysms of the brachial artery following venipuncture in infants. Pediatr Surg Int 2004;20:594-7.

26. Li S, Sharma P, Gupta N, Abdelbaki A. Got Wire? A Case of Retained Guidewire During Midline Venous Catheter Placement and How to Prevent It. Connecticut Med 2017;81:555-9.

27. Rutkoff GS. The influence of an antimicrobial peripherally inserted central catheter on central line-associated bloodstream infections in a hospital environment. J Assoc Vascular Access 2014;19:172-9. 